Objective This prospective study examined the prevalence of dyslipidaemia in postpartum women diagnosed with gestational diabetes mellitus (GDM).

Methods Women with GDM were reviewed 6-8 weeks postpartum. A fasting lipid profile was performed. Clinical details were recorded from the medical records, including the woman's weight and body mass index (BMI) measured at her first antenatal visit.

Results Of the 98 women studied, the mean age was 33.0 years (range $23-45$ years) and $30.6 \%(n=31)$ were primigravid. The mean BMI was $30.6 \mathrm{~kg} / \mathrm{m}^{2}$ and $52 \%(\mathrm{n}=51)$ were obese. The overall prevalence of dyslipidaemia was $52 \%(n=51)$. Total cholesterol was raised in $44 \%(n=43)$, low-density lipoprotein was raised in 33\% ( $n=32)$ and triglycerides were raised in $16 \%(\mathrm{n}=16)$. Of the 51 women with dyslipidaemia, $73 \%(n=37)$ had more than one abnormality in their lipid profile. The prevalence of dyslipidaemia was $78 \%(n=14)$ in women with moderate to severe obesity $\left(B M I>34.5 \mathrm{~kg} / \mathrm{m}^{2}\right) \mathrm{com}-$ pared with $50 \%(n=22)$ in non-obese women $(p<0.0001)$. Of the 5 women with an abnormal GTT postpartum, $80 \%(n=4)$ had an abnormal lipid profile.

Conclusion Women with an abnormal GTT in pregnancy should be screened for dyslipidaemia postpartum at the time of their repeat GTT, and if the lipids are abnormal they should be offered cardioprotective interventions and ongoing monitoring of their lipid profile.

\section{PM.06 LOW MOLECULAR HEPARIN WITHIN THE UTEROPLACENTAL UNIT}

doi:10.1136/archdischild-2013-303966.091

'SK Ismail, ' 2 L Norris, 'L Kelly, 'JR Higgins. 'Anu Research Centre, Department of Obstetrics and Gynaecology, University College Cork, Cork University Maternity Hosp, Cork, Ireland; ' $C$ Coagulation Research Laboratory, Department of Obs and Gynae, Trinity Centre for Health Sciences, St. James Hospital, Dublin, Ireland

Background Perturbation of the uteroplacental haemostasis has been implicated in placenta mediated pregnancy complications in thrombophilic women. LMWH may be effective in altering local thrombin production in the uteroplacental compartment.

Aim We determined the effects of LMWH (tinzaparin) on the peripheral, uteroplacental and fetal circulation and on haemostatic gene and antigen expression in placental tissue.

Method Eight women on antenatal LMWH prophylaxis (tinzaparin $75 \mathrm{IU} / \mathrm{kg}$ ) due to moderate risk of VTE undergoing caesarean section (CS) and a control group of 15 healthy pregnant women undergoing CS had venous blood taken from the peripheral and uterine vein before delivery of placenta. Simultaneously, cord venous blood and placental biopsy was collected. Tissue factor pathway inhibitor (TFPI), thrombin antithrombin (TAT) and endogenous thrombin potential (ETP) were measured. Real-time PCR and ELISA were used to quantify mRNA and protein expression of TFPI and TF in placental tissue.

Results TAT levels within uterine vein are significantly higher compared to maternal peripheral circulation in both the control group $(\mathrm{P}<0.0001)$ and LMWH group $(\mathrm{P}<0.02)$. In the LMWH group, TAT is reduced compared with controls in the uterine vein $(\mathrm{P}<0.001)$. ETP and TFPI within uterine circulation is reduced significantly in the LMWH group $(\mathrm{P}<0.05)$ and $(\mathrm{P}<0.02)$ respectively. Down-regulation of placental TFPI and TFPI 2 mRNA expression was also found $(p<0.05)$. Placental TF mRNA expression in LMWH group showed a non significant increase compared to control and this is replicated in placental TF antigen expression.

Conclusion TAT is reduced in uteroplacental circulation in thrombophilic women on LMWH prophylaxis and this is mirrored by decreased ETP in uteroplacental circulation. LMWH may be effective in reducing in-vivo thrombin production in the uteroplacental circulation of thrombophilic women.

\section{PM.07 A PROSPECTIVE STUDY OF CHANGES IN MATERNAL CARDIOVASCULAR AND METABOLIC FUNCTION FROM PRIOR TO PREGNANCY TO POSTPARTUM}

doi:10.1136/archdischild-2013-303966.092

${ }^{1} \mathrm{AA}$ Mahendru, ${ }^{1} \mathrm{TR}$ Everett, ${ }^{2} \mathrm{CM}$ McEniery, ${ }^{2} \mathrm{~B}$ Wilkinson, ${ }^{1} \mathrm{CC}$ Lees. ${ }^{1}$ Fetal Medicine Department, Addenbrooke's Hospital, Cambridge University Hospital NHS Foundation Trust, Cambridge, UK; ${ }^{2}$ Clinical Pharmacology Department, University of Cambridge, Cambridge, UK

Cardiovascular adaptation in normal pregnancy is the key to understanding cardiovascular function in pregnancy complications. The objective of this study was to investigate changes in maternal cardiovascular function during pregnancy, from a pre-pregnancy baseline to the postpartum period.

In this prospective study, 54 women had normal pregnancy outcome; 5 had preeclampsia (PE) and/or intrauterine growth insufficiency (IUGR). Detailed haemodynamics were assessed prepregnancy, at median gestation of 6, 23 and 33 weeks and 16 weeks postpartum. Lipid profile and renal function were assessed pre-pregnancy, in first trimester and postpartum.

While heart rate (HR) increased throughout pregnancy $(P=0.001)$, brachial and central BP, together with peripheral vascular resistance (PVR) and wave reflections were reduced very early in pregnancy $(P<0.001)$, followed by an increase in third trimester. Cardiac output (CO) increased to a peak by second trimester $(P=0.001)$. The $\mathrm{HR}, \mathrm{CO}$ and $\mathrm{PVR}$ returned to pre-pregnancy values in the postpartum period. However, the reduction in BP was sustained postpartum. The MAP increased in second trimester rather than a decrease in women with PE/IUGR $(P=0.02)$. Lipids and creatinine decreased in first trimester $(P<0.001)$.

This is the first study to investigate longitudinal changes in central BP and wave reflections from pre-pregnancy to postpartum. We demonstrated profound changes in BP and arterial wave reflections very early in pregnancy; however $\mathrm{CO}$ peaks in the second trimester. The reduction in BP below pre-pregnancy values was sustained postpartum. Prospective studies of cardiovascular adaptation, beginning from pre-pregnancy are more likely to provide reliable estimates of pregnancy related maternal cardiovascular changes.

\section{PM.08 METFORMIN, GLYCAEMIC CONTROL AND POSTNATAL GLUCOSE-TOLERANCE-TESTING IN WOMEN WITH GESTATIONAL DIABETES}

doi:10.1136/archdischild-2013-303966.093

LI Stirrat, H Mustafa, CDB Love. Department of Obstetrics, Royal Infirmary of Edinburgh, EDINBURGH, UK

Background Metformin has been shown to be safe, effective and acceptable to women with gestational diabetes (GDM), and is recommended as the first-line pharmacological treatment for women who meet the usual criteria for insulin.

Methods We retrospectively reviewed case notes of women with GDM in the Royal Infirmary of Edinburgh (RIE) from January 2009-March 2011. Audit standards derived from local guidelines included: metformin as the first-line glucose-lowering medication in at least $90 \%$ of cases; average blood glucose readings $<8.0 \mathrm{mmol} / \mathrm{L}$ over two weeks in at least $90 \%$ of cases $(\geq 8.0 \mathrm{mmol} / \mathrm{L}$ was considered 'poor' glycaemic control); and postnatal glucose-tolerance-test (GTT) in all cases. Neonatal outcomes were observed.

Results Of the 113 pregnancies reviewed, 82.3\% (93/113) of women required glucose-lowering medication. Metformin was used first-line in $94.6 \%$ of women requiring medication (88/93), and $99.6 \%$ of these continued treatment until delivery. Supplemental insulin was required in $44.3 \%$ of cases (39/88). Average blood glucose readings of $<8.0 \mathrm{mmol} / \mathrm{L}$ were achieved in $93.6 \%$, and $91.2 \%$ of cases during the second and third trimesters respectively. $70.8 \%$ of 